\title{
Effects of oestrogen deficiency on the alveolar bone of rats with experimental periodontitis
}

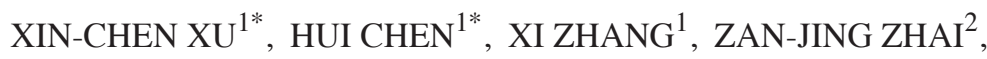 \\ XU-QIANG LIU ${ }^{2}$, XIN-YI ZHENG ${ }^{1}$, JUN ZHANG ${ }^{2}, \mathrm{AN} \mathrm{QIN}^{2}$ and ER-YI LU ${ }^{1}$ \\ ${ }^{1}$ Department of Prosthodontics, Shanghai Key Laboratory of Stomatology; ${ }^{2}$ Department of Orthopaedics, \\ Shanghai Key Laboratory of Orthopaedic Implants, Shanghai Ninth People's Hospital, \\ Shanghai Jiao Tong University School of Medicine, Shanghai 200011, P.R. China
}

Received August 14, 2014; Accepted May 1, 2015

DOI: $10.3892 / \mathrm{mmr} .2015 .3875$

\begin{abstract}
Periodontitis is an inflammatory disease characterized by loss of connective tissue and alveolar bone, and osteoporosis is a common disease characterized by a systemic impairment of bone mass and microarchitecture. To date, the association between periodontitis and osteoporosis has remained to be fully elucidated. In the present study, an experimental rat model of periodontitis was used to explore the effects of oestrogen deficiency-induced osteoporosis on the maxillary alveolar bone. Forty-four female, six-month-old Sprague-Dawley rats were randomly divided into four groups: Control, ligature, ovariectomized (OVX), and OVX + ligature. One month after ovariectomy, rats in the ligature and OVX + ligature groups received ligatures on their first and second maxillary molars for 1 month. Fluorescent labelling was performed prior to sacrificing the animals. At the end of the experiment, the maxillae and serum were collected and subjected to micro-computed tomography analysis, confocal laser-scanning microscopic observation, Van Gieson's fuchsin staining, tartrate-resistant acid phosphatase staining and ELISA. Ligatures slightly reduced the alveolar bone mineral density (BMD) and bone formation rate, but significantly
\end{abstract}

Correspondence to: Dr Jun Zhang or Dr An Qin, Department of Orthopaedics, Shanghai Key Laboratory of Orthopaedic Implants, Shanghai Ninth People's Hospital, Shanghai Jiao Tong University School of Medicine, 639 Zhizaoju Road, Shanghai 200011, P.R. China

E-mail: zhangjun2@medmail.com.cn

E-mail:dr.qinan@gmail.com

Dr Er-Yi Lu, Department of Prosthodontics, Shanghai Key Laboratory of Stomatology, Shanghai Ninth People's Hospital, Shanghai Jiao Tong University School of Medicine, 639 Zhizaoju Road, Shanghai 200011, P.R. China

E-mail: lueryi222@126.com

*Contributed equally

Key words: osteoporosis, periodontitis, ovariectomy, alveolar bone reduced alveolar crest height $(\mathrm{ACH})$. Ovariectomy reduced the alveolar BMD, impaired the trabecular structure, reduced the bone formation rate and increased the serum levels of bone resorption markers. Animals in the OVX + ligature group exhibited a lower alveolar BMD, a poorer trabecular structure, a reduced $\mathrm{ACH}$, a lower bone formation rate and higher serum levels of bone resorption markers compared with those in the control group. The results of the present study showed that ovariectomy enhanced alveolar bone loss and reduced the $\mathrm{ACH}$ of rats with experimental periodontitis. Thus, post-menopausal osteoporosis may influence the progression of periodontitis.

\section{Introduction}

Periodontitis is defined as a bacteria-induced disease that gradually destroys periodontal tissues, including the gums, cementum, periodontal ligaments and supporting alveolar bone $(1,2)$. Data from 2009-2010 showed that almost half of the US population over 30 years of age $(47.2 \%)$ suffered from a certain degree of periodontitis, including $8.7 \%$ with mild disease, $30.0 \%$ with moderate disease and $8.5 \%$ with severe periodontitis (3). Due to the high prevalence of periodontitis and accompanying loss of teeth or the edentulous jaw, most elderly people regard oral health as an important aspect of life quality for various physical, social and psychological reasons (4).

Osteoporosis is a common disease characterized by systemic bone loss and impaired bone microarchitecture. Post-menopausal women are usually more vulnerable to osteoporosis due to their decreased oestrogen levels that affect bone metabolism $(5,6)$. Guiglia et al $(7)$ described a possible association between osteoporosis and periodontal disease, suggesting that osteoporosis may facilitate the alveolar bone resorption caused by periodontitis (7). Specifically, osteoporosis results in an increase in certain inflammatory factors, a number of which also participate in the progression of periodontitis.

In recent decades, numerous studies have focused on the association between osteoporosis and periodontitis at the bone level. Several studies have reported that osteoporosis promotes the loss of periodontal attachment, loss of alveolar bone height and even tooth loss (8-10). Tezal et al (11) reported that low skeletal bone mineral density (BMD) is associated 
with loss of proximal alveolar bone and clinical attachment. By contrast, several studies have suggested that this association was weak (12), and Brennan-Calanan et al (13) reported that systemic bone density and oral infection independently influence oral bone loss in post-menopausal women. However, previous studies have also shown a great diversity in sample sizes and measurement methodologies, and most have been retrospective clinical studies (14-17). Thus, whether oestrogen deficiency-induced systemic bone loss jeopardizes alveolar bone remains controversial.

A small number of well-controlled experimental animal studies have investigated the association between osteoporosis and periodontitis. Through histometric analyses and assessment of serum alkaline phosphatase and calcium, Duarte et al (18) demonstrated that oestrogen-deficiency may significantly increase bone loss resulting from ligature-induced periodontitis in rats, and that there was a synergistic effect between oestrogen deficiency and plaque accumulation. Amadei et al (19) observed a significant increase in bone loss, morphometrically evaluated using photo documentation, when ligation occurred 90 days after rats underwent ovariectomy, suggesting that long-term oestrogen deficiency affects ligature-induced alveolar bone loss. However, other studies were unable to correlate the absence of ovarian hormones with periodontal alterations in rats using radiographic analyses with digital dental X-ray equipment (20). In the present study, using micro-computed tomography (micro-CT) analysis, the effect of oestrogen deficiency-induced osteoporosis on the alveolar bone of rats with experimental periodontitis (EP) was explored. It was hypothesized that oestrogen deficiency-induced osteoporosis not only facilitates alveolar bone loss, but also jeopardizes bone microarchitecture in local alveolar bone.

\section{Materials and methods}

Animals, treatments and experimental design. Forty-four female, six-month-old Sprague-Dawley rats with a mean weight of $400 \pm 30 \mathrm{~g}$ were obtained from the Department of Laboratory Animal Science (Ninth People's Hospital, Shanghai Jiao Tong University School of Medicine, Shanghai, China). The rats were housed in a $21^{\circ} \mathrm{C}$ room with a 12 -h light/dark cycle. The general condition of the animals was monitored daily and body weight was recorded weekly. The Ethics Committee and the Animal Care and Use Committee of Shanghai Jiao Tong University School of Medicine (Shanghai, China) approved the experimental protocol and the procedures performed.

After two weeks of adaptation, the rats were randomly divided into four groups, with 11 rats in each group: The control, ligature, OVX and OVX + ligature groups. Rats in the OVX and OVX + ligature groups underwent bilateral ovariectomy, whereas the other animals underwent a sham surgery $(21,22)$. Four weeks later, EP was induced by placing 3/0 silk sutures (Johnson \& Johnson Medical, Shanghai, China) subgingivally around the bilateral first and second maxillary molars (M1 and M2, respectively) for four weeks. Ligature placement initiated local inflammation and alveolar crest bone resorption $(23,24)$. To ensure the establishment of $\mathrm{EP}$, the ligatures were checked twice weekly and replaced when necessary. All treatments were conducted under general anaesthesia (10\% chloral hydrate; Sigma-Aldrich, St. Louis, $\mathrm{MO}, \mathrm{USA} ; 4 \mathrm{ml} / \mathrm{kg}$ via intraperitoneal injection).

Each rat was injected intraperitoneally with tetracycline (TE; $25 \mathrm{mg} / \mathrm{g}$ ), alizarin red (AL; $20 \mathrm{mg} / \mathrm{kg}$ ), and calcein (CA, $10 \mathrm{mg} / \mathrm{kg}$ ) (all from Sigma-Aldrich) at 25, 15, and 5 days, respectively, prior to sacrification. At the designated end-point, blood samples were collected by cardiac puncture under anaesthesia and centrifuged (6,500-7,000 x g, 15-20 min) to recover the serum, which was stored at $-80^{\circ} \mathrm{C}$ until analysis. Subsequently, the rats were sacrificed with an overdose of anaesthesia. Bilateral maxillary bone specimens were harvested, fixed in 4\% paraformaldehyde (Sigma-Aldrich) for $48 \mathrm{~h}$ and transferred to $70 \%$ ethanol prior to further testing.

Micro-CT scanning and assessment of the alveolar crest height (ACH). A cone-beam micro-CT system (Skyscan1176; Skyscan, Kontich, Belgium) at Soochow University Orthopaedic Institute (Suzhou, China) was used to scan maxillary bone specimens. The X-ray generator was set at a voltage of $50 \mathrm{KV}$, a current of $500 \mu \mathrm{A}$ and a fixed shutter speed of $900 \mathrm{msec}$. The images were re-constructed using NRecon (version 1.5.1.4; Skyscan, Kontich, Belgium). Fig. 1A illustrates the region of interest (ROI) for analysis of tooth-supporting alveolar bone in the maxillae. On the basis of a selected Hounsfield Unit (HU) grayscale threshold value, microstructural indicators of BMD, bone volume/tissue volume ratio (BV/TV), trabecular thickness (Tb.Th), trabecular separation (Tb.Sp), trabecular number (Tb.N), structure-model index (SMI) and connectivity density (Conn.Dn) were calculated using CTAn (version 1.10; Skyscan). These measures were used to quantify the bone (BV/TV), determine the average width of the bone structure (Tb.Th), determine the number of traversals across the bone trabeculae per unit length (Tb.N), determine the distance between trabeculae that corresponds to the bone marrow measurement (Tb.Sp), determine the number of trabecular elements that can be removed without changing the bone network and provide an estimate for the number of trabecular connections per $\mathrm{mm}^{3}$ (Conn.Dn), and indicate the relative prevalence of rod-like or plate-like trabecular bone (SMI). SMI was defined as an interval between 0 and 3 , where 0 is an ideal plate-like structure and 3 is a cylinder.

According to a previous study (25), the linear distance between the cement-enamel junction $(\mathrm{CEJ})$ and alveolar bone crest $(\mathrm{ABC})$ was measured to reflect the decreased volume of alveolar crest height $(\mathrm{ACH})$. The decreased $\mathrm{ACH}$ was obtained by averaging the CEJ-ABC distances measured at the mesiolingual, mesiobuccal, distolingual and distobuccal parts of the specimen. Clinically, a decreased $\mathrm{ACH}$ illustrates the integrity of tooth-supporting alveolar bone, with lower CEJ-ABC values reflecting better-quality alveolar bone (26).

Bone histomorphometry. The left maxillae were sequentially de-hydrated, de-calcified, embedded in methyl methacrylate (Sigma-Aldrich). Sections were prepared from the occlusal surface of the tooth crown to the alveolar bone mesiodistally along the plane parallel to the long axis of the tooth and then cut to 100-200 $\mu \mathrm{m}$ using the Leica SP1600 Microtome (Leica Microsystems, Heidelberg, Germany) and polished to a final thickness of approximately $20 \mu \mathrm{m}$ by sequential usage 
A

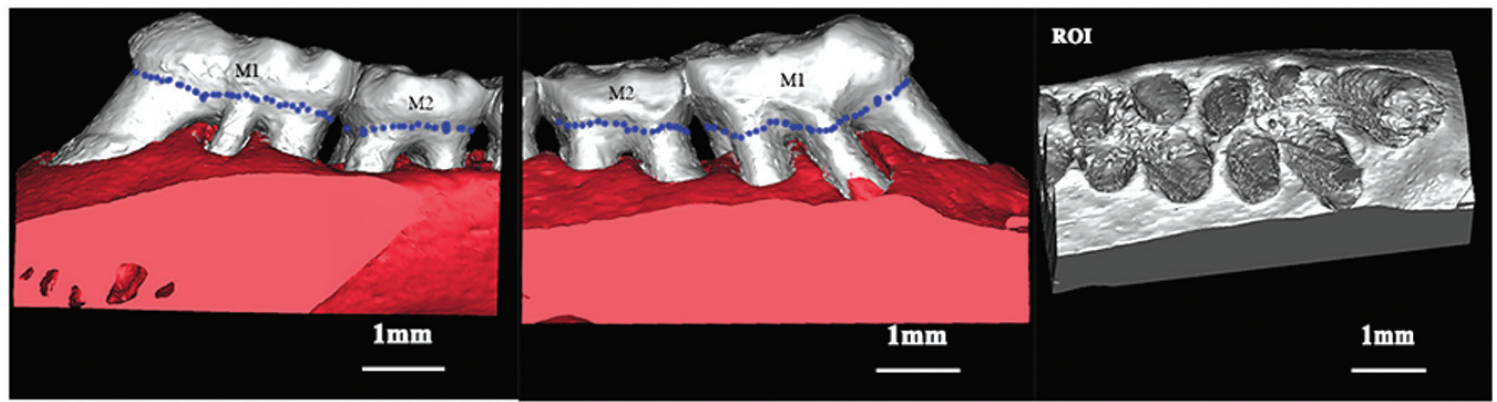

B

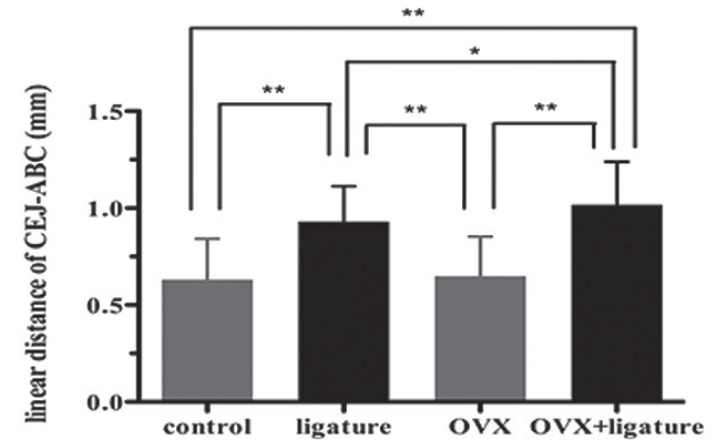

D

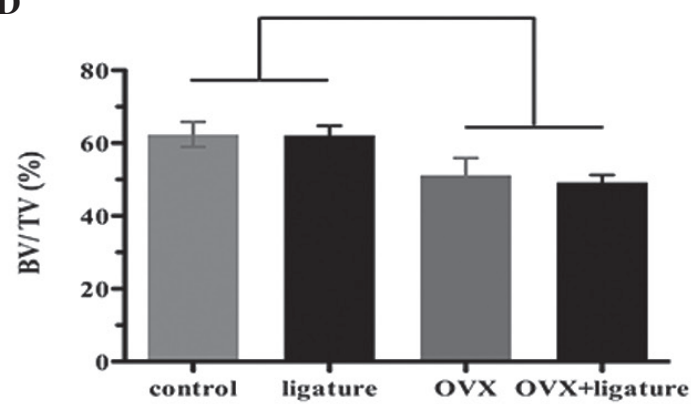

F

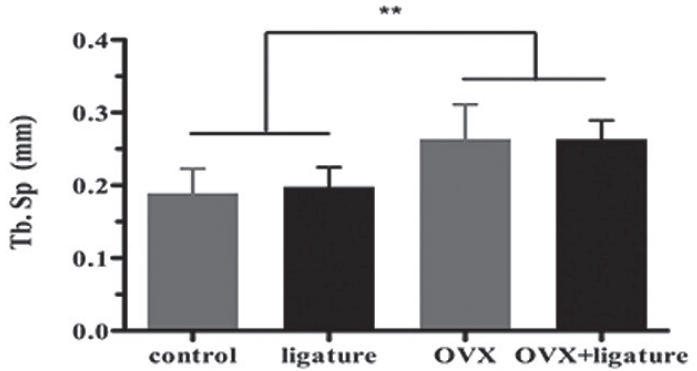

$\mathbf{H}$

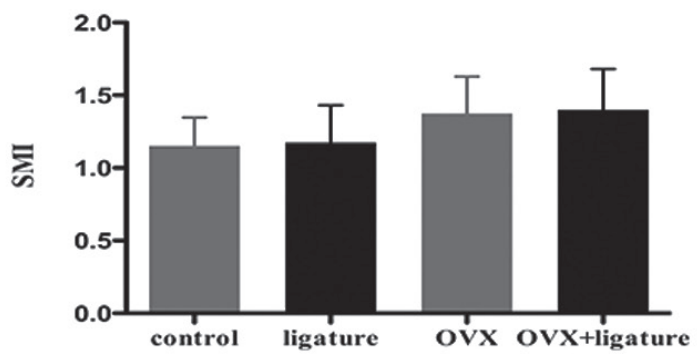

C

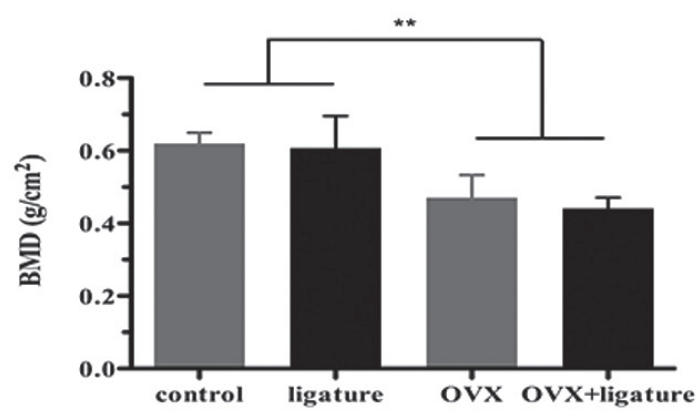

$\mathbf{E}$

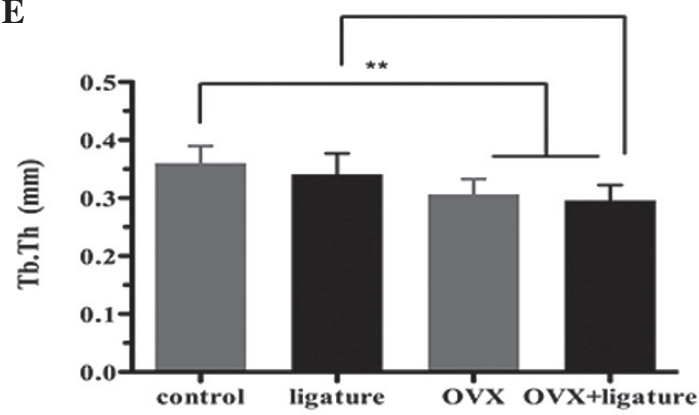

G

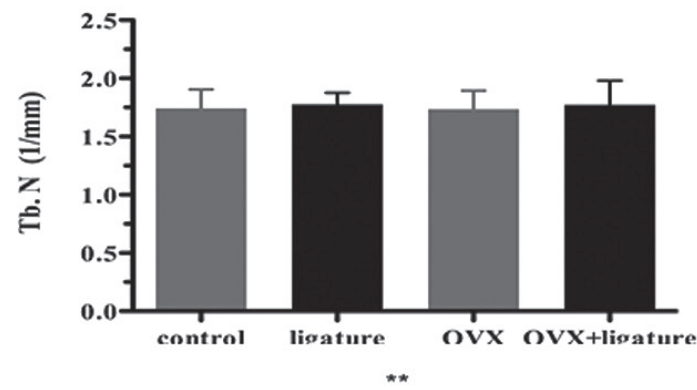

I

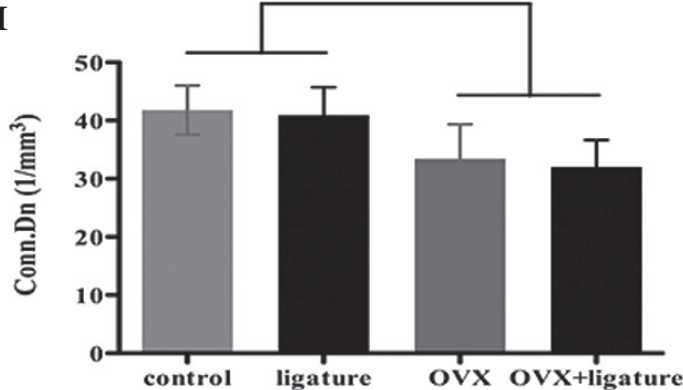

Figure 1. Effects of ovariectomy on $\mathrm{ACH}$ and alveolar bone microarchitecture of rats with experimental periodontitis by micro-computed tomography analysis. (A) Buccal and palatal sides of the maxillary alveolar bone, where the $\mathrm{ACH}$ was measured between the $\mathrm{CEJ}$ and the $\mathrm{ABC}$ in the mesiolingual, mesiobuccal, distolingual and distobuccal regions of the M1 and M2. The ROI is a cuboidal bone body that encompasses the M1 and M2 roots. (B) Analysis of the CEJ-ABC linear distance after 1 month of ligation. (C-I) Analysis of micro-computed tomography volumetric parameters: (C) BMD, (D) BV/TV ratio, (E) Tb.Th, (F) Tb.Sp, (G) Tb.N, (H) SMI and (I) Conn.Dn. Values are expressed as the mean \pm standard deviation $\left({ }^{*} \mathrm{P}<0.05\right.$; $\left.{ }^{* *} \mathrm{P}<0.01\right)$. OVX, ovariectomy; ACH, alveolar crest height; CEJ, cement-enamel junction; ABC, alveolar bone crest; ROI, region of interest; M1, first maxillary molar; M2, second maxillary molar; BMD, bone mineral density; BV/TV, bone volume/tissue volume; Tb.Th, trabecular thickness; Tb.Sp, trabecular separation; Tb.N, trabecular number; SMI, structure-model index; Conn.Dn, connectivity density. 


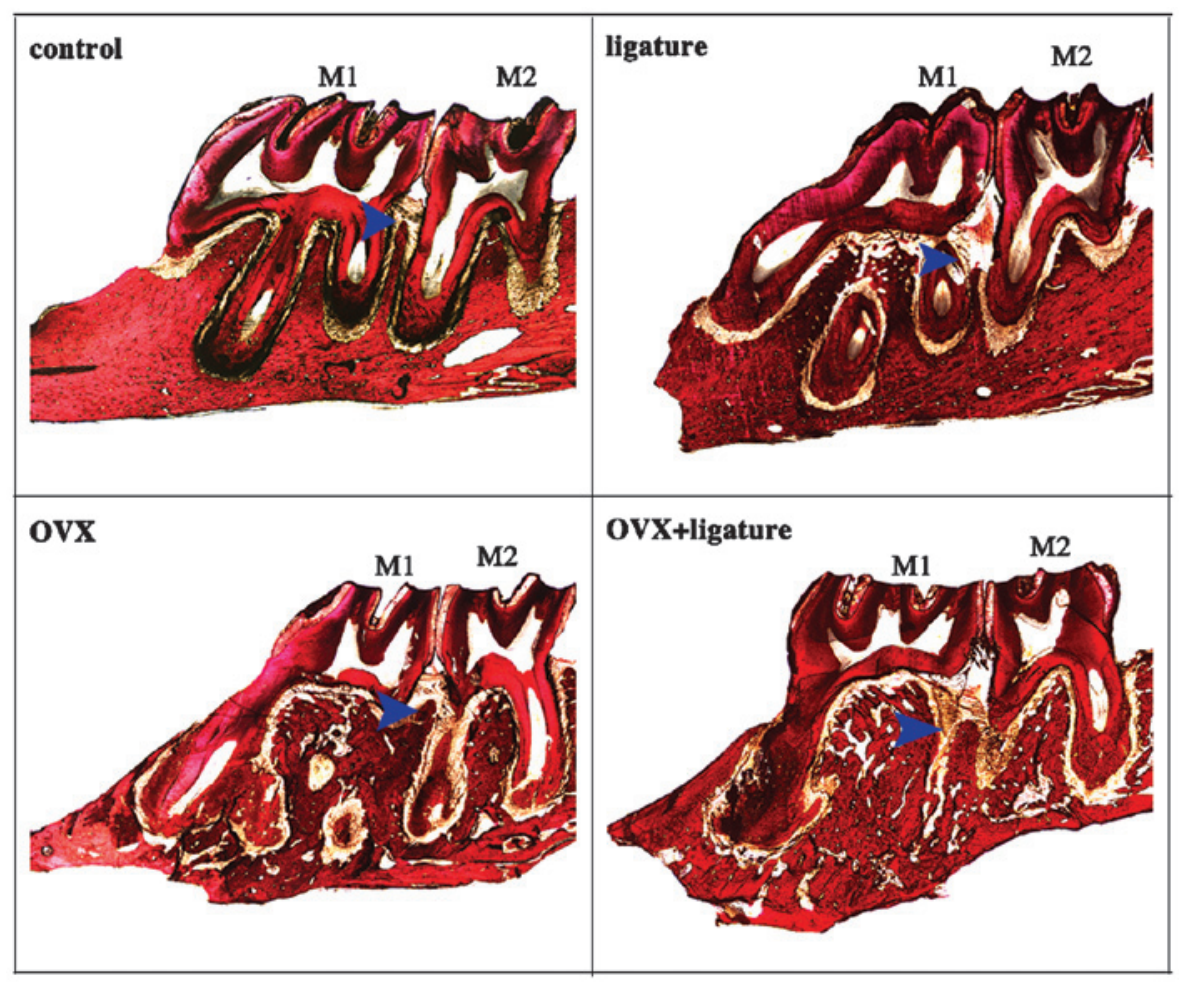

Figure 2. Effect of OVX on alveolar bone in rats with experimental periodontitis, based on a histomorphometric analysis. Images of the maxillary bone surrounding the M1 and M2 after staining with Van Gieson's fuchsin (magnification, x40). Blue arrows show the alveolar crest in these longitudinal sections. OVX, ovariectomy; M1, first maxillary molar; M2, second maxillary molar.

of P300, P800 and P1200 sandpaper. To obtain the mineral apposition rate (MAR), fluorescent labeling, which was performed in the live rats as described above, was visualized. A confocal laser-scanning microscope (TCS Sp2 AOBS; Leica Microsystems, Wetzlar, Germany) was used to capture images of the fluorescent labeling line (27). The excitation/emission wavelengths for each of the fluorochromes were 405/580 nm (TE; yellow), 543/617 nm (AL; red) and 488/517 nm (CA; green). Sections were also stained with Van Gieson's fuchsin (Sigma-Aldrich) for histological observation. The tabecular bone dynamic parameters were measured from a $1 \mathrm{~mm}^{2}$ box positioned at the interradicular region of M1 (magnification, x100), using tetracycline and calcein labels. MAR was measured using a Bioquant image analysis system (Bioquant OSTEO II Version 8.12.20, Bioquant Image Analysis Corporation, Nashville, TN, USA).

The right maxillae were de-calcified in $10 \%$ EDTA (Sigma-Aldrich) for 2 months and then embedded in paraffin. Serial sagittal sections $(5 \mu \mathrm{m})$ were stained with osteoclast-specific, tartrate-resistant acid phosphatase (TRAP; Sigma-Aldrich), observed microscopically (Eclipse 90i; Nikon, Tokyo, Japan) and micrographed. ImageJ 1.46r software (National Institutes of Health, Bethesda, MD, USA) was used to analyze the TRAP- and Van Gieson's fuchsin-stained sections. The number of TRAP-positive, multinucleated osteoclasts was counted in each sample.

Serum bone resorption biomarkers. The serum bone-specific resorption markers, tartrate-resistant acid phosphatase $5 \mathrm{~b}$ (TRACP5b) and C-terminal telopeptide of type I collagen (CTX-1), were quantified in all serum samples using rat
ELISA kits (SB-TR102 and AC-06F1; IDS, Fountain Hills, AZ, USA).

Statistical analysis. All values are expressed as the mean \pm standard deviation. One-way analysis of variance with Bonferroni's post hoc test was performed to compare the between-group means for all outcomes. The Statistical Package for the Social Sciences, version 17.0 (SPSS, Inc., Chicago, IL, USA) was used for all analyses. $\mathrm{P}<0.05$ or $\mathrm{P}<0.01$ was considered to indicate a statistically significant difference between groups.

\section{Results}

Effect of ovariectomy on ACH. To obtain ACH values, the linear CEJ-ABC distances at four sites for each specimen were measured and averaged (Fig. 1). The ACH decreased by an average of $0.2979 \mathrm{~mm}$ in the ligature group and by $0.3858 \mathrm{~mm}$ in the OVX + ligature group, compared with that in the control animals (both $\mathrm{P}<0.001$; Fig. 1B). Compared to that in the OVX group, the $\mathrm{ACH}$ decreased by $0.2814 \mathrm{~mm}$ in the ligature group and by $0.3693 \mathrm{~mm}$ in the OVX + ligature group $(\mathrm{P}<0.001$; Fig. 1B). Statistical analysis of the comparison between the control and OVX groups indicated that ovariectomy did not have any effect on the $\mathrm{ACH}$ of non-ligature rats. However, ovariectomy promoted alveolar bone resorption, affecting the $\mathrm{ACH}$ in the rats in the $\mathrm{OVX}+$ ligature group, as indicated by the lower $\mathrm{ACH}$ in the $\mathrm{OVX}+$ ligature group compared with that in the ligature group ( $\mathrm{P}=0.042$; Fig. 1B). Furthermore, an obvious $\mathrm{ACH}$ decrease in the ligature group and in the OVX + ligature group was observed between M1 and M2 in the Van Gieson's fuchsin-stained sections (Fig. 2; blue arrow). 

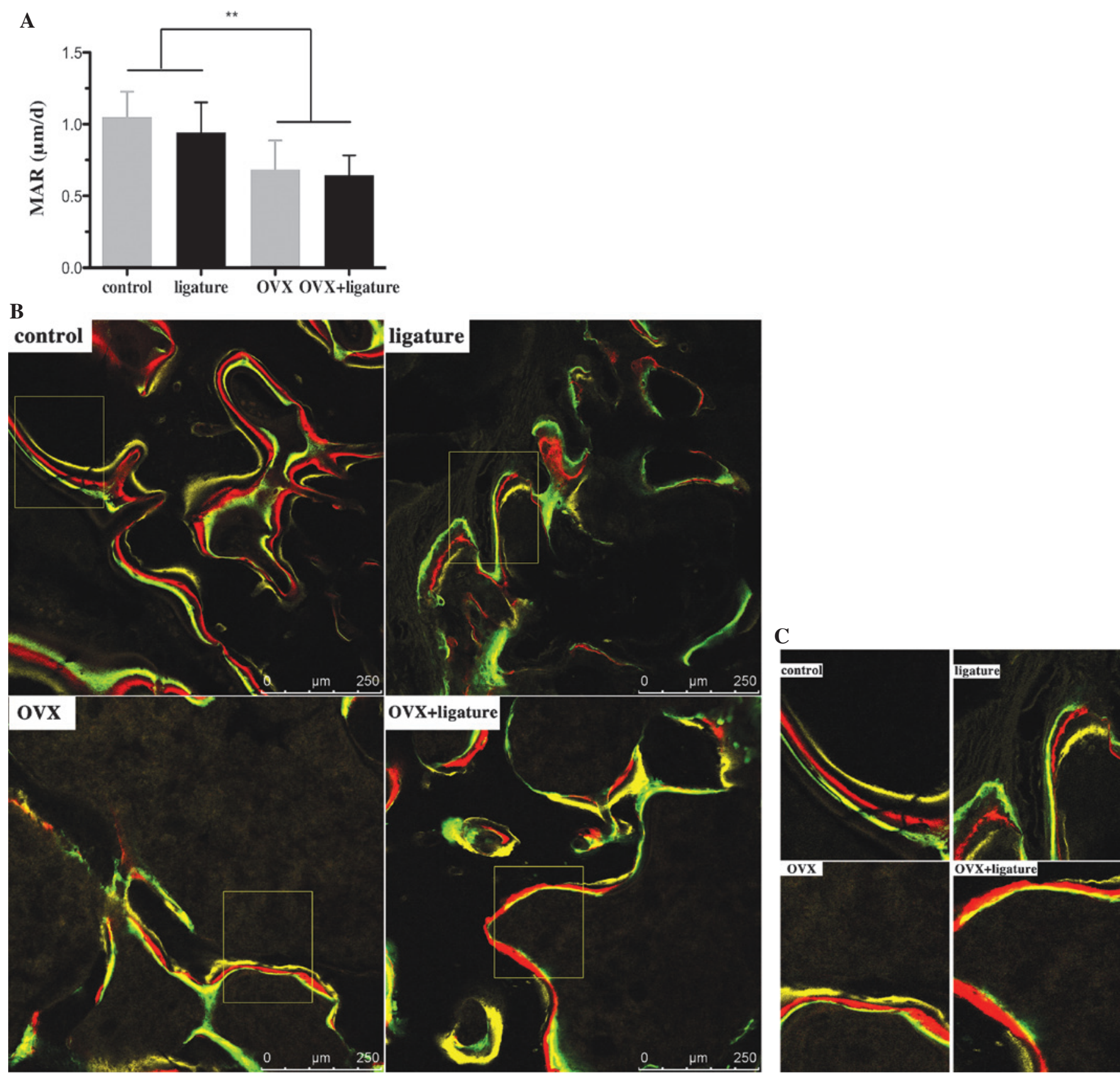

Figure 3. Effect of OVX on the MAR in rats with experimental periodontitis, based on fluorescent labelling. (A) Analysis of MAR. Images of fluorescent labeling was captured using a confocal laser-scanning microscope and MAR was measured using a Bioquant image analysis system with a $1 \mathrm{~mm}^{2}$ box positioned in the inter-radicular region of $\mathrm{M} 1$ at a magnification of $\mathrm{x} 100$, using tetracycline and calcein labels $\left({ }^{* *} \mathrm{P}<0.01\right)$. (B) Images of sequential fluorescent labelling of the alveolar bone, showing the results of tetracycline (yellow), alizarin red (red) and calcein (green) staining (magnification, x100). (C) Magnified excerpts of B indicated by boxes (magnification, x200). The space between two labels represents newly mineralized bone that developed during the 10-day period between label injections. MAR, bone mineral apposition rate; OVX, ovariectomy.

Effect of ovariectomy on alveolar bone microarchitecture. Based on the analysis of the ROI in the alveolar bone, OVX rats exhibited lower BMDs than non-OVX rats $(\mathrm{P}<0.001$; Fig. 1C). A decreased BV/TV ratio was associated with a reduced $\mathrm{Tb}$.Th as well as an increased $\mathrm{Tb} . \mathrm{Sp}$ in the OVX and OVX + ligature groups compared with those in the control and ligature groups $(\mathrm{P}<0.001$; Fig. 1D-F). There was also a significant decrease in the Conn.Dn in the OVX and OVX + ligature groups, whereas the Tb.N and SMI remained relatively consistent among the groups (Fig. 1G-I). Fig. 2 shows the bone profile in a longitudinal section. Obvious oste- olysis was detected in the OVX rats, which was not present in the ligature-only group.

Effect of ovariectomy on alveolar bone MAR and the number of active osteoclasts. Due to the oestrogen deficiency resulting from ovariectomy, significantly lower MAR values were observed in the OVX rats compared with those in the non-OVX rats $(\mathrm{P}<0.001$; Fig. 3A). EP mildly aggravated the trend, as indicated by the slightly lower MAR value in the OVX + ligature animals than that in the OVX-only group. The yellow, red and green fluorescent labelling lines were intertwined in the 


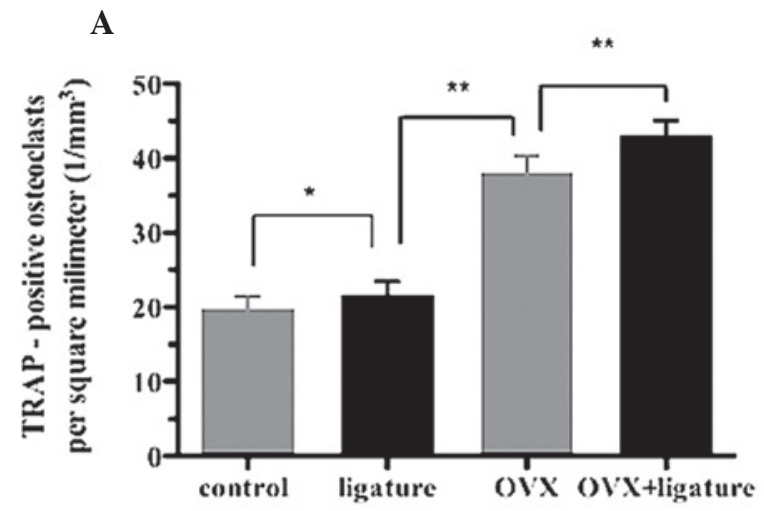

B

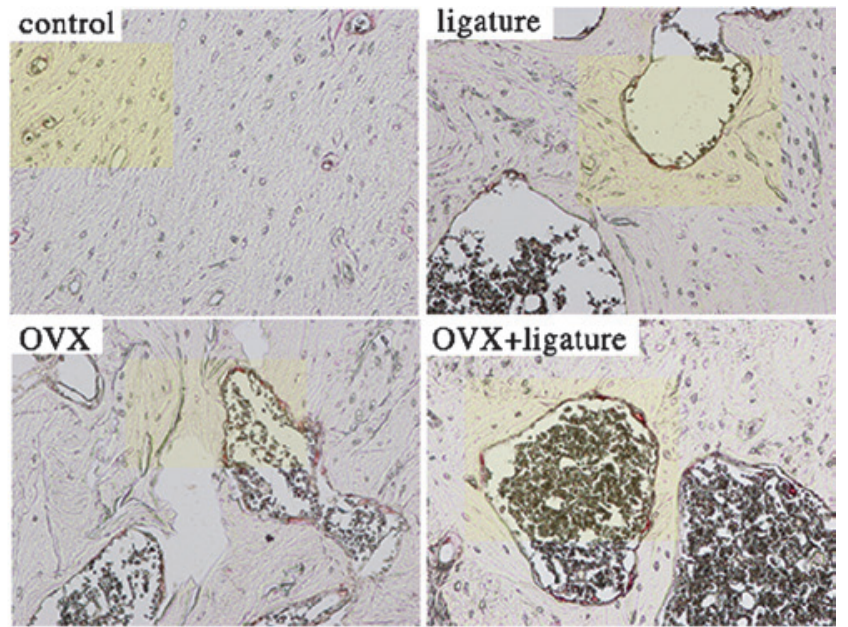

C
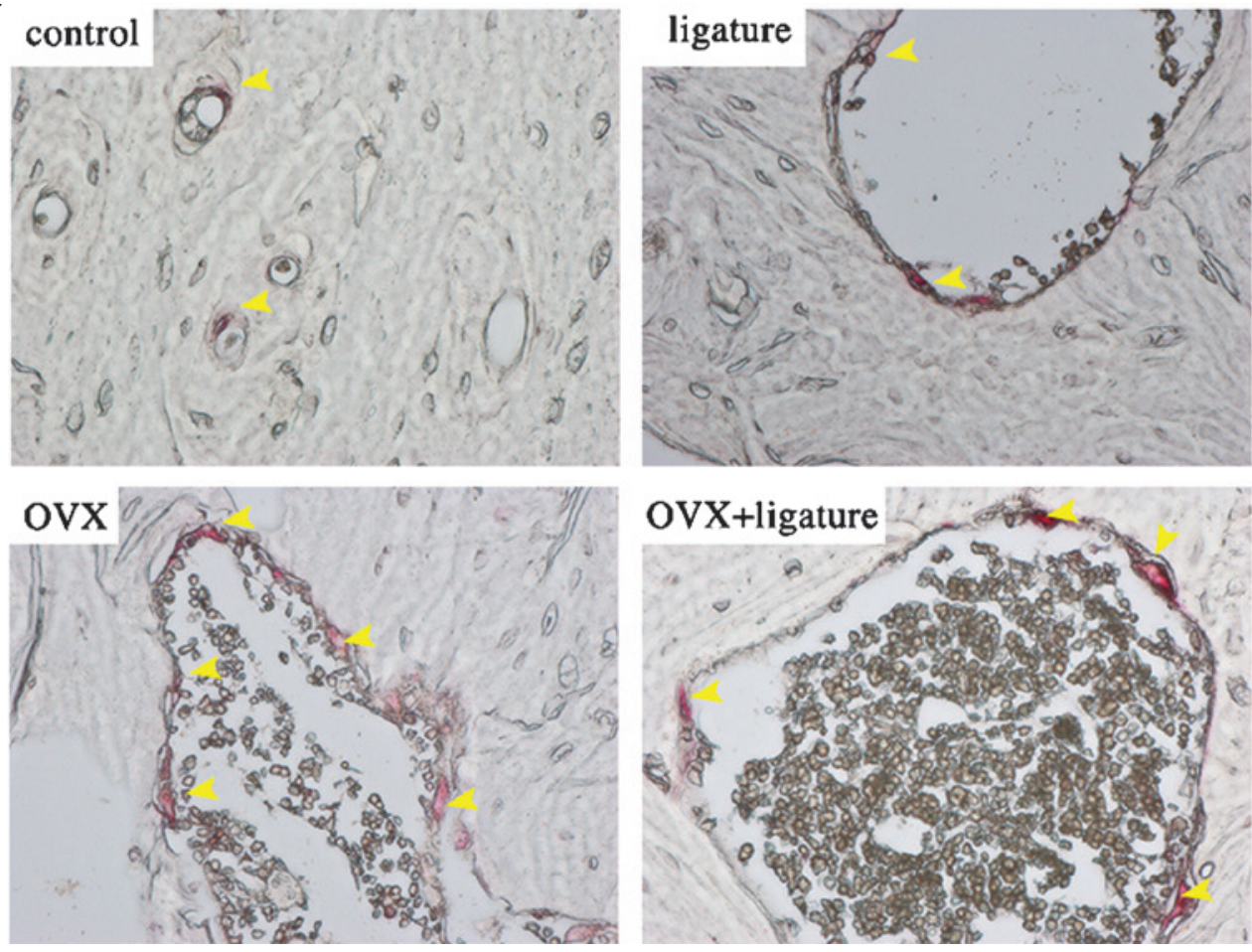

Figure 4. Effect of OVX on osteoclast numbers and activity in rats with experimental periodontitis, based on TRAP staining. (A) Analysis of the number of osteoclasts in a $1-\mathrm{mm}^{2}$ area. Values are expressed as the mean \pm standard deviation $\left({ }^{*} \mathrm{P}<0.05 ;{ }^{* *} \mathrm{P}<0.01\right)(\mathrm{B})$ Images of alveolar bone after TRAP staining (magnification, x200). (C) Magnified areas from B highlighted in a yellow (magnification, x400). Multinuclear osteoclasts are stained red (yellow arrows). TRAP, tartrate-resistant acid phosphatase; OVX, ovariectomy.

OVX + ligature group, in contrast to the obvious separation between the lines in the control group (Fig. 3B and C).

Analysis of the TRAP-stained sections showed that the number of osteoclasts was increased in the ligature, OVX and OVX + ligature groups by $9.59,92.42$ and $117.42 \%$, respectively, compared to that in the control group (Fig. 4A). In addition, variations in the shape and location of the osteoclasts were also found among the four groups. In contrast to the control and ligature groups, the OVX group and particularly the OVX + ligature group exhibited larger, irregularly shaped osteoclasts and more eroded bone surfaces (Fig. 4B and C; arrows).

Effect of ovariectomy on serum levels of TRACP5b and CTX-1. The OVX + ligature group showed elevated serum TRACP5b levels as compared with those in the $\mathrm{OVX}(\mathrm{P}=0.017)$, ligature
$(\mathrm{P}<0.001)$ and control $(\mathrm{P}<0.001)$ groups, as well as elevated serum CTX-1 levels compared to those in the ligature $(\mathrm{P}=0.001)$ and control $(\mathrm{P}<0.001)$ groups. In addition, the OVX group showed increased serum TRACP5b levels compared to those in the ligature $(\mathrm{P}=0.921)$ and control $(\mathrm{P}=0.030)$ groups, as well as increased serum CTX-1 levels compared with those in the ligature $(\mathrm{P}=0.001)$ and control $(\mathrm{P}<0.001)$ groups. Furthermore, the ligature group showed significantly higher serum CTX-1 levels $(\mathrm{P}<0.001)$ and slightly higher TRACP5b levels $(\mathrm{P}=0.839)$ compared with those in the control group (Fig. 5).

\section{Discussion}

Extensive epidemiologic and experimental studies have proven the existence of systemic risk factors pertaining to the 
A

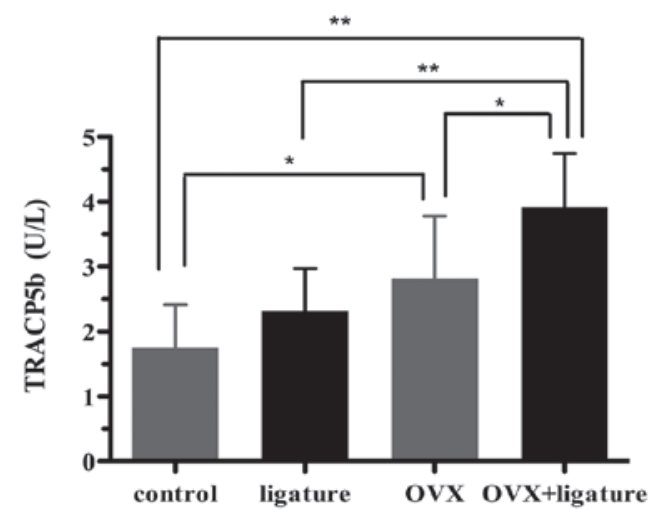

B

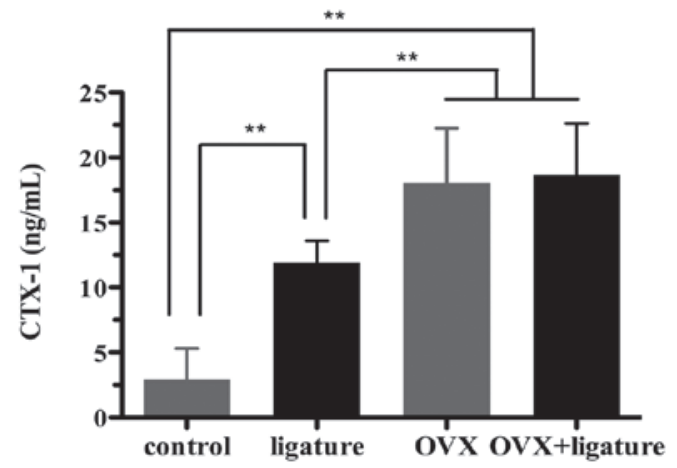

Figure 5. Effect of OVX on the serum biochemical markers (A) TRACP5b and (B) CTX-1 in rats with experimental periodontitis. TRACP5b and CTX-1 were quantified using ELISA and the values are expressed as the mean \pm standard deviation ( $\left.{ }^{*} \mathrm{P}<0.05 ;{ }^{* *} \mathrm{P}<0.01\right)$. TRACP5b, tartrate-resistant acid phosphatase $5 \mathrm{~b}$; CTX-1, C-terminal telopeptide of type I collagen; OVX, ovariectomy.

initiation, progression and severity of periodontitis $(28,29)$. A novel concept also recognizes that systemic risk factors may determine the rate of progression, age at onset and severity of periodontal disease (30). Among these risk factors, osteoporosis is one of the six main factors (30). In addition, periodontitis and osteoporosis are major health problems among the elderly (31). Therefore, an explicit understanding of the correlation between the two diseases is critical to the prevention of the morbidity and mortality associated with the disorders, particularly in the elderly. However, additional clarification regarding the association between osteoporosis and periodontitis is still required, in addition to an understanding of the extent to which osteoporosis contributes to the overall risk of periodontitis. To better understand these associations, experimental animal models are valuable for initial investigations. Sex steroid deficiency, particularly that of oestrogen, is considered to be the main cause of osteoporosis, and OVX rats are widely used as animal models of osteoporosis $(18,20,32)$. In addition, the periodontal tissue of rats resembles that of humans (33).

Alveolar bone is an important component of dental treatments, including prosthodontic, implant and orthodontic treatments $(31,34)$. When analyzing bone mass and volumetric bone parameters, the present study identified that ovariectomy had a significant influence on all parameters except Tb.N and SMI, whereas local simulation of periodontitis failed to significantly change these parameters. This observation was in accordance with a previous study (19), which showed that liga- tion at 90 days after ovariectomy induced a significant increase in bone loss compared with that in the ligature only group. However, ligatures concurrent with ovariectomy only slightly aggravated the alveolar bone loss, compared with that following ovariectomy alone. The $\mathrm{ACH}$ is a measure for the evaluation of periodontitis $(9,13)$. In the present study, ovariectomy alone did not reduce the $\mathrm{ACH}$, but aggravated the reduction in $\mathrm{ACH}$ in combination with ligatures. Low alveolar BMD and impaired alveolar bone structure may lead to increased bone resorption of the alveolar crest when periodontitis occurs concurrently with ovariectomy. In addition, ovariectomy-induced oestrogen deficiency disturbs the cytokines that may increase host susceptibility to infection, which also facilitates the progression of periodontitis (31,35-37).

The coupling of bone resorption and bone formation is of great significance to bone metabolism, which is affected by hormones and cytokines (38). In the present study, the bone formation rate was lower than the bone resorption rate in OVX rats, in contrast to the balanced parameters in non-OVX rats, which could be observed from the MAR rate. In contrast to the control and ligature groups, the MAR rate decreased in the OVX and OVX + ligature groups, while there was no statistical difference between the control group and ligature group. The mixed fluorescent labelling line and the reduced MAR of the OVX rats indicated that oestrogen deficiency-induced osteoporosis influenced the balance of alveolar bone formation and resorption. However, no significant MAR reduction was observed in the ligature rats, which may be due the time required for bacterial accumulation prior to impacting bone metabolism after ligation. The local accumulation of bacteria and bacteria-derived factors may stimulate a local inflammatory reaction and activation of the innate immune system. The immune cells secrete cytokines that promote osteoclast maturation, leading to an imbalance in bone metabolism (1). However, these processes take time to occur. Therefore, rats in the OVX + ligature group showed the lowest MAR among the four groups due to the synergic effect. In the present study, the number of TRAP-positive osteoclasts demonstrated an increasing trend in the ligature, OVX and OVX + ligature groups. However, ligature-induced periodontitis led to a significantly increased number of osteoclasts. Furthermore, the levels of two specific serum bone resorption markers, TRACP5b and CTX-1, increased in the ligature rats, but not in the OVX rats. Interleukin (IL)-6 and IL-1 in bone marrow cells are known to stimulate osteoclastic bone resorption under conditions of oestrogen deficiency (39).

Periodontitis is a disease normally caused by bacterial infection, which produces factors or antigens that stimulate local inflammatory reactions and activities of the innate immune system (1). Previous studies have suggested that cytokines, including IL-17 or tumour necrosis factor- $\alpha$ (TNF- $\alpha$ ), have a key role in periodontal alveolar bone resorption (40-43). IL-17 exerts its osteoclastogenic activity by enhancing receptor activator of nuclear factor kappa-B ligand expression in osteoblasts and $\mathrm{CD}^{+} \mathrm{T}$ cells, and promotes alveolar resorption when released in excessive amounts (44-46). TNF- $\alpha$ directly contributes to periodontal damage through its effect on osteoclastogenesis, through amplification of inflammatory immune reactions and through the inhibition of differentiation and bone nodule formation (47-49). Thus, oestrogen deficiency, associated with increased systemic levels of IL-17 or TNF- $\alpha$, 
may augment the local levels of these factors in the alveolar bone and facilitate alveolar bone resorption. This concept is in accord with previously presented results that suggested low oestrogen induced T- and B-cell abnormalities, increased local production of the bone-active cytokines, and finally resulted in periodontitis progression (50).

In conclusion, the present study demonstrated that ovariectomy resulted in the deterioration of the alveolar bone microarchitecture, $\mathrm{ACH}$ reduction, decline in the bone formation rate and increased osteoclast activity. These observations indicated that post-menopausal osteoporosis impacts the progression of periodontitis.

\section{Acknowledgements}

Grant support for this project was provided by Shanghai Leading Academic Discipline Project (nos. T0202 and S30206) and the National College Students' Innovative Entrepreneurial Training Plan (no. 2013053). The authors would like to thank Dr. Tingting Tang, who provided advice on osteoporosis, as well as Drs Qiming Fan, Shuhong Zhang and Shuangyan Zhang, (Department of Orthopaedics, Shanghai Ninth People's Hospital, Shanghai Jiao Tong University School of Medicine, Shanghai, China), who provided experimental guidance, and Dr Junjie Lu, who provided great assistance with the animal surgeries (Department of SPF laboratory, Shanghai Ninth People's Hospital, Shanghai Jiao Tong University School of Medicine).

\section{References}

1. Di Benedetto A, Gigante I, Colucci S and Grano M: Periodontal disease: linking the primary inflammation to bone loss. Clin Dev Immunol 2013: 503754, 2013.

2. Pihlstrom BL, Michalowicz BS and Johnson NW: Periodontal diseases. Lancet 366: 1809-1820, 2005.

3. Eke PI, Dye BA, Wei L, Thornton-Evans GO and Genco RJ: CDC Periodontal Disease Surveillance workgroup: James Beck GDRP: Prevalence of periodontitis in adults in the United States: 2009 and 2010. J Dent Res 91: 914-920, 2012.

4. McGrath C and Bedi R: The importance of oral health to older people's quality of life. Gerodontology 16: 59-63, 1999.

5. Nordin BE, Wishart JM, Clifton PM, et al: A longitudinal study of bone-related biochemical changes at the menopause. Clin Endocrinol (Oxf) 61: 123-130, 2004.

6. NIH Consensus Development Panel on Osteoporosis Prevention, Diagnosis, and Therapy: Osteoporosis prevention, diagnosis, and therapy. JAMA 285: 785-795, 2001.

7. Guiglia R, Di Fede O, Lo Russo L, Sprini D, Rini GB and Campisi G: Osteoporosis, jawbones and periodontal disease. Med Oral Patol Oral Cir Bucal 18: e93-e99, 2013.

8. Yoshihara A, Seida Y, Hanada N and Miyazaki H: A longitudinal study of the relationship between periodontal disease and bone mineral density in community-dwelling older adults. J Clin Periodontol 31: 680-684, 2004.

9. Wactawski-Wende J, Hausmann E, Hovey K, Trevisan M, Grossi S and Genco RJ: The association between osteoporosis and alveolar crestal height in postmenopausal women. J Periodontol 76 (11 Suppl): 2116-2124, 2005.

10. Ronderos M, Jacobs DR, Himes JH and Pihlstrom BL: Associations of periodontal disease with femoral bone mineral density and estrogen replacement therapy: cross-sectional evaluation of US adults from NHANES III. J Clin Periodontol 27: 778-786, 2000.

11. Tezal M, Wactawski-Wende J, Grossi SG, Ho AW, Dunford R and Genco RJ: The relationship between bone mineral density and periodontitis in postmenopausal women. J Periodontol 71 1492-1498, 2000.

12. Lundström A, Jendle J, Stenström B, Toss G and Ravald N: Periodontal conditions in 70-year-old women with osteoporosis. Swed Dent J 25: 89-96, 2001
13. Brennan-Calanan RM, Genco RJ, Wilding GE, Hovey KM, Trevisan $\mathrm{M}$ and Wactawski-Wende $\mathrm{J}$ : Osteoporosis and oral infection: independent risk factors for oral bone loss. J Dent Res 87: 323-327, 2008

14. Aspalli SS, Shetty VS, Parab PG, Nagappa G, Devnoorkar A and Devarathnamma MV: Osteoporosis and periodontitis: Is there a possible link? Indian J Dent Res 25: 316-320, 2014.

15. Lin TH, Lung CC, Su HP, et al: Association between periodontal disease and osteoporosis by gender: A nationwide population-based cohort study. Medicine (Baltimore) 94: e553, 2015.

16. Moeintaghavi A, Pourjavad M, Dadgar S and Tabbakh NS: Evaluation of the association between periodontal parameters, osteoporosis and osteopenia in post menopausal women. J Dent (Tehran) 10: 443-448, 2013.

17. Hattatoglu-Sonmez E, Ozcakar L, Gokce-Kutsal Y, Karaagaoglu E, Demiralp B and Nazliel-Erverdi H: No alteration in bone mineral density in patients with periodontitis. J Dent Res 87: 79-83, 2008.

18. Duarte PM, Gonçalves PF, Sallum AW, Sallum EA, Casati MZ and Humberto Nociti F Jr: Effect of an estrogen-deficient state and its therapy on bone loss resulting from an experimental periodontitis in rats. J Periodontal Res 39: 107-110, 2004.

19. Amadei SU, Souza DM, Brandão AA and Rocha RF: Influence of different durations of estrogen deficiency on alveolar bone loss in rats. Braz Oral Res 25: 538-543, 2011.

20. Anbinder AL, Prado Mde A, Spalding M, et al: Estrogen deficiency and periodontal condition in rats: a radiographic and macroscopic study. Braz Dent J 17: 201-207, 2006.

21. Thompson DD, Simmons HA, Pirie CM and Ke HZ: FDA guidelines and animal models for osteoporosis. Bone 17: 125S-133S, 1995

22. Liu XL, Li CL, Lu WW, Cai WX and Zheng LW: Skeletal site-specific response to ovariectomy in a rat model: change in bone density and microarchitecture. Clin Oral Implants Res 26: 392-398, 2015.

23. Cai X, Li C, Du G and Cao Z: Protective effects of baicalin on ligature-induced periodontitis in rats. J Periodontal Res 43: 14-21, 2008.

24. Breivik T, Opstad PK, Gjermo P and Thrane PS: Effects of hypothalamic-pituitary-adrenal axis reactivity on periodontal tissue destruction in rats. Eur J Oral Sci 108: 115-122, 2000.

25. Park CH, Abramson ZR, Taba M Jr, et al: Three-dimensional micro-computed tomographic imaging of alveolar bone in experimental bone loss or repair. J Periodontol 78: 273-281, 2007.

26. Hu KF, Ho YP, Ho KY, Wu YM, Wang WC and Chou YH: Clinical case report on treatment of generalized aggressive periodontitis: 5-year follow-up. Int J Periodontics Restorative Dent 35: 395-400, 2015.

27. Jiang XQ, Wang SY, Zhao J, Zhang XL and Zhang ZY: Sequential fluorescent labeling observation of maxillary sinus augmentation by a tissue-engineered bone complex in canine model. Int J Oral Sci 1: 39-46, 2009.

28. Kornman KS: Mapping the pathogenesis of periodontitis: A new look. J Periodontol 79: 1560-1568, 2008.

29. Borrell LN and Papapanou PN: Analytical epidemiology of periodontitis. J Clin Periodontol 32 (Suppl 6): 132-158, 2005.

30. Genco RJ and Borgnakke WS: Risk factors for periodontal 2000 disease. Periodontol 62: 59-94, 2013

31. Wactawski-Wende J, Grossi SG, Trevisan M, et al: The role of osteopenia in oral bone loss and periodontal disease. J Periodontol 67 (10 Suppl): 1076-1084, 1996.

32. Sultan N and Rao J: Association between periodontal disease and bone mineral density in postmenopausal women: a cross sectional study. Med Oral Patol Oral Cir Bucal 16: e440-447, 2011.

33. Page RC, Offenbacher S, Schroeder HE, Seymour GJ and Kornman KS: Advances in the pathogenesis of periodontitis: summary of developments, clinical implications and future directions. Periodontol 2000 14: 216-248, 1997.

34. Hirai T, Ishijima T, Hashikawa Y and Yajima T: Osteoporosis and reduction of residual ridge in edentulous patients. J Prosthet Dent 69: 49-56, 1993.

35. Golden SH, Robinson KA, Saldanha I, Anton B and Ladenson PW: Clinical review: Prevalence and incidence of endocrine and metabolic disorders in the United States: A comprehensive review. J Clin Endocrinol Metab 94: 1853-1878, 2009.

36. Hildebolt CF, Pilgram TK, Yokoyama-Crothers N, et al: The pattern of alveolar crest height change in healthy postmenopausal women after 3 years of hormone/estrogen replacement therapy. J Periodontol 73: 1279-1284, 2002. 
37. Payne JB, Reinhardt RA, Nummikoski PV and Patil KD Longitudinal alveolar bone loss in postmenopausal osteoporotic/osteopenic women. Osteoporos Int 10: 34-40, 1999.

38. Wronski TJ, Dann LM, Scott KS and Cintron M: Long-term effects of ovariectomy and aging on the rat skeleton. Calcif Tissue Int 45: 360-366, 1989.

39. Miyaura C, Kusano K, Masuzawa T, et al: Endogenous bone-resorbing factors in estrogen deficiency: cooperative effects of IL-1 and IL-6. J Bone Miner Res 10: 1365-1373, 1995

40. Cardoso CR, Garlet GP, Crippa GE, et al: Evidence of the presence of $\mathrm{T}$ helper type 17 cells in chronic lesions of human periodontal disease. Oral Microbiol Immunol 24: 1-6, 2009.

41. Vernal R, Dutzan N, Chaparro A, Puente J, Antonieta Valenzuela $\mathrm{M}$ and Gamonal J: Levels of interleukin-17 in gingival crevicular fluid and in supernatants of cellular cultures of gingival tissue from patients with chronic periodontitis. J Clin Periodontol 32: 383-389, 2005.

42. Graves D: Cytokines that promote periodontal tissue destruction. J Periodontol 79 (8 Suppl): 1585-1591, 2008.

43. Garlet GP, Martins W Jr, Fonseca BA, Ferreira BR and Silva JS Matrix metalloproteinases, their physiological inhibitors and osteoclast factors are differentially regulated by the cytokine profile in human periodontal disease. J Clin Periodontol 31: 671-679, 2004
44. Yu JJ, Ruddy MJ, Wong GC, et al: An essential role for IL-17 in preventing pathogen-initiated bone destruction: recruitment of neutrophils to inflamed bone requires IL-17 receptor-dependent signals. Blood 109: 3794-3802, 2007.

45. Boyle WJ, Simonet WS and Lacey DL: Osteoclast differentiation and activation. Nature 423: 337-342, 2003

46. Weaver CT, Harrington LE, Mangan PR, Gavrieli M and Murphy KM: Th17: an effector CD4 T cell lineage with regulatory T cell ties. Immunity 24: 677-688, 2006.

47. Tjoa ST, de Vries TJ, Schoenmaker T, Kelder A, Loos BG and Everts V: Formation of osteoclast-like cells from peripheral blood of periodontitis patients occurs without supplementation of macrophage colony-stimulating factor. J Clin Periodontol 35: $568-575,2008$

48. Yarilina A, Xu K, Chen J and Ivashkiv LB: TNF activates calcium-nuclear factor of activated T cells (NFAT)c1 signaling pathways in human macrophages. Proc Natl Acad Sci USA 108: $1573-1578,2011$.

49. Huang H, Zhao N, Xu X, et al: Dose-specific effects of tumor necrosis factor alpha on osteogenic differentiation of mesenchymal stem cells. Cell Prolif 44: 420-427, 2011.

50. Inagaki K, Kurosu Y, Sakano M, et al: Osteoporosis and periodontal disease in postmenopausal women: Association and mechanisms. Clin Calcium 16: 269-277, 2006 (In Japanese). 